\title{
Phytochemical Analysis Revealing the Presence of Two New Compounds from the Latex of Calotropis Procera (Ait.) R.Br.
}

\author{
Hiren V. Doshi ${ }^{1{ }^{1 *} \text {, Farzin M. Parabia }}{ }^{1}$, Falguni K. Sheth ${ }^{2}$, Indravadan L. Kothari ${ }^{3}$, Minoo H. Parabia ${ }^{2}$, \\ Arabinda Ray ${ }^{4}$ \\ ${ }^{1}$ Ashok \& Rita Patel Institute of Integrated Study and Research In Biotechnology \& Allied Sciences (ARIBAS), \\ New Vallabh Vidyanagar, 388121, India \\ ${ }^{2}$ Rasiklal Manikchand Dhariwal Ayurvedic Hospital and College, Vagaldhara, Valsad, 396375, India \\ ${ }^{3}$ Department of Bioscience, Sardar Patel University, Vallabh Vidyanagar, 388120, India \\ ${ }^{4}$ P D Patel Institute of Applied Sciences (PDCP), Charotar University of Science and Technology (CHARUSAT), Changa, 388421, India
}

\begin{abstract}
Calotropis procera (Ait.) R.Br. (Asclepiadaceae) is a well known Indian medicinal plant. Its latex constituents having high calorific value because of its rich hydrocarbons. Moreover it was traditionally applied for tanninizing leather in India. Several attempts have been made in the past to establish it as a biofuel crop. In the present study the major phyto-constituents from the dried latex of $C$. procera are identified through GC-MS analysis. Acetone extract derived from the dried latex of $C$. procera was further partitioned between n-hexane and ethanol (10:90). The n-hexane layer was separated, dried and re-dissolved in Acetone. The un-dissolved part was dissolved in n-Hexane:Methanol (9:1) combination. Both the fractions were subjected for GC-MS analysis and nine hydrocarbon compounds have been identified from the latex, out of which 2,6 dimethyl tetra-1,5-decaene and 3, 7,11-Trimethyl-2,6,10,12-pentadecatrien-1-ol are newly reported from latex of C. procera.
\end{abstract}

Keywords Calotropis procera, Asclepiadaceae, medicinal plant, bio-energy, latex, phytochemistry, hydrocarbons, GC-MS

\section{Introduction}

Calotropis procera belongs to the family Asclepiadaceae and is a well known Indian medicinal plant locally known as "Akado". Medicinally important parts of this plant are flower, terminal leaf pairs, root with root bark and latex. Out of that the most significant part which drew attention of researchers is latex because of its medicinal properties, economic use for cheese and rubber production. Latex contain majority of hydrocarbon contents[1]. Kumar et al.[2] have established this plant as a source of bio-energy. The plant also shows good antibacterial activity[3]. Therefore, in the present study the major latex constituents were separated and identified through GC-MS analysis.

\section{Material and Methods}

\subsection{Plant Material}

* Corresponding author:

drhirendoshi@yahoo.co.in (Hiren V. Doshi)

Published online at http://journal.sapub.org/plant

Copyright (C) 2012 Scientific \& Academic Publishing. All Rights Reserved
Latex of $C$. procera was collected from the arid area of Siddhpur, North Gujarat, India. The latex was dried in oven at $42^{\circ} \mathrm{C}$ to obtain $20 \%$ dry powder.

\subsection{Extraction and Characterization}

The dry latex powder was extracted with acetone in Soxhlet apparatus for $12 \mathrm{hrs}$. The dried acetone extract was partitioned between $n$-hexane and 90\% ethanol combined in 1:5 ratios. The layer of $n$-hexane was separated and evaporated to get oily mixture of hydrocarbons in liquid form. All hydrocarbons were separated through TLC in solvent system n-hexane: di-ethyl ether: acetic acid (80:20:1). The resolved bends compared with the standard compounds oleic acid, triolein and $\beta$-sitosterol. The TLC plate developed with the spray of vanillin-sulphuric acid[4]. The dried n-hexane layer was again re-dissolved in acetone and remaining part was dissolved in $\mathrm{n}$ - hexane:methanol (9:1) in warm condition $\left(60^{\circ} \mathrm{C}\right)$. Both the fractions of $n$-hexane extract were analyzed by GC-MS. Solvents used for extraction and purification were from MERCK company.

\subsection{Parameters of GC-MS Analysis}

GC-MS model: Perkin Elmer Autosystem XL with Turbomass, Column type: PE-5MS, Column Material: 5\% 
Phenyl polysiloxane, Column Length: 30 meters, Column inner diameter: $0.250 \mathrm{~mm}$, Flow rate $\left(\mathrm{N}_{2}\right): 1 \mathrm{ml} / \mathrm{min}$, Temperature of injector: $230^{\circ} \mathrm{C}$, Temperature of detector: $280^{\circ} \mathrm{C}$, Temperature of source: $280^{\circ} \mathrm{C}$, Temperature of transfer: $280^{\circ} \mathrm{C}$, Programming rate: Starting from $78^{\circ} \mathrm{C}$ for $5 \mathrm{~min}$. Increasing temperature with rate $10^{\circ} \mathrm{C} / \mathrm{min}$ up to $280^{\circ} \mathrm{C}$ and hold for $20 \mathrm{~min}$. Retention time: $45 \mathrm{~min}$

\section{Result and Discussion}

Latex of Calotropis procera is well known for cardiac glycosides and hydrocarbons [5]. The reported cardiac glycosides were Calotropogenin[6], Calotropin[7], Calotoxin, Uscharin and Calactin[8] with identification of some hydrocarbon derivatives like Linoleic acid, Oleic acid and Palmitic acid[9].

The maximum portion of dry latex of $C$. procera extracted by acetone was $54 \%$. During initial screening the other solvents tried were n-hexane, petroleum ether, chloroform and dimethyl sulphoxide but none of them could extract as much large part as acetone. Majority of the latex is composed of hydrocarbons therefore n-hexane part of acetone extract was concentrated for separation and identification. The presence of three marker compounds oleic acid (Rf: 0.51 ), triolein (Rf: 0.89 ) and $\beta$-sitosterol (Rf: 0.30 ) were confirmed through TLC.

Major nine compounds of $n$-hexane fraction are identified from both the fractions of $n$-hexane as described in experimental section through GC-MS. The identified compounds are checked with the available references and the two compounds are not found to be reported anywhere from C. procera are 2,6 dimethyl tetra-1,5-decaene and 3, 7,11-Trimethyl-2,6,10,12-pentadecatrien-1-ol, they are reported as the new phytoconstituents from $C$. procera. The four another compounds are excluded from the study are Ethyl phthalate, Di-n-Propyl phthalate, Phthalic acid diisobutyl ester and Phthalic acid diisoheptyl ester as the contaminants. Their details of retention time and relative percentage of the corresponding latex components are given in Tables-1, $2 \& 3$.

The electron impact mass spectral (EI-MS) fragmentations of the major nine prominent phytochemicals from the latex of $C$. procera are narrated as under:

Table 1. Major chemical constitution from latex of $C$. procera

\begin{tabular}{|c|c|c|c|}
\hline Compound & $\begin{array}{c}\text { RT } \\
(\mathbf{m i n})\end{array}$ & $\begin{array}{c}\text { Conc. } \\
\mathbf{( \% )}\end{array}$ & Identified compound \\
\hline 1. & 3.569 & 1.16 & 4-hydroxy-4-methylpentan-2-one \\
\hline 2. & 4.467 & 1.09 & 2,3,4-trimethylhexane \\
\hline 3. & 7.015 & 3.71 & Decane \\
\hline 4. & 12.772 & 1.16 & n-Pentadecane \\
\hline 5. & 16.861 & 1.93 & 2,6 dimethyl tetra-1,5-decaene \\
\hline 6. & 24.928 & 1.17 & n-Eicosane \\
\hline 7. & 32.371 & 5.98 & $\begin{array}{c}3,7,11 \text {-Trimethyl-2,6,10,12 } \\
\text {-pentadecatrien-1-ol }\end{array}$ \\
\hline$* 8$. & 32.390 & 0.80 & $\begin{array}{c}2,6,10,15,19,23-H e x a m e t h y l-2,6, \\
10,14,18,22-t e t r a c o s a h e x a e n e\end{array}$ \\
\hline 9. & 35.341 & 17.54 & $1,3,5-$ Triisopropylbenzene \\
\hline
\end{tabular}

Table 2. Major chemical constitution from latex of $C$. procera

\begin{tabular}{|c|c|c|c|c|}
\hline $\begin{array}{c}\text { Com- } \\
\text { pound }\end{array}$ & $\begin{array}{c}\text { M.W. } \\
\text { (g/mol) }\end{array}$ & $\begin{array}{c}\text { Molecular } \\
\text { Formula }\end{array}$ & CAS Number & $\begin{array}{c}\text { Boiling } \\
\text { Point }\left({ }^{\circ} \mathbf{C}\right)\end{array}$ \\
\hline 1. & 116.16 & $\mathrm{C}_{6} \mathrm{H}_{12} \mathrm{O}_{2}$ & $000123-42-2$ & 116 \\
\hline 2. & 128.26 & $\mathrm{C}_{9} \mathrm{H}_{20}$ & $000921-47-1$ & 140 \\
\hline 3. & 142.28 & $\mathrm{C}_{10} \mathrm{H}_{22}$ & $000124-18-5$ & 174 \\
\hline 4. & 212.42 & $\mathrm{C}_{15} \mathrm{H}_{32}$ & $000629-62-9$ & 270 \\
\hline 5. & 220.40 & $\mathrm{C}_{16} \mathrm{H}_{28}$ & ---- & -- \\
\hline 6. & 282.56 & $\mathrm{C}_{20} \mathrm{H}_{42}$ & $000112-95-8$ & 220 \\
\hline 7. & 262.44 & $\mathrm{C}_{18} \mathrm{H}_{30} \mathrm{O}$ & ---- & -- \\
\hline$* 8$. & 410.72 & $\mathrm{C}_{30} \mathrm{H}_{50}$ & $000111-02-4$ & 285 \\
\hline 9. & 204.35 & $\mathrm{C}_{15} \mathrm{H}_{24}$ & $000717-74-8$ & 232 \\
\hline
\end{tabular}

*Peak No 8 from n-hexane:methanol (9:1) soluble part of n-hexane layer of acetone extract from dry Calotropis procera latex.

Table 3. Structural configuration of major chemical constitution from latex of Calotropis procera

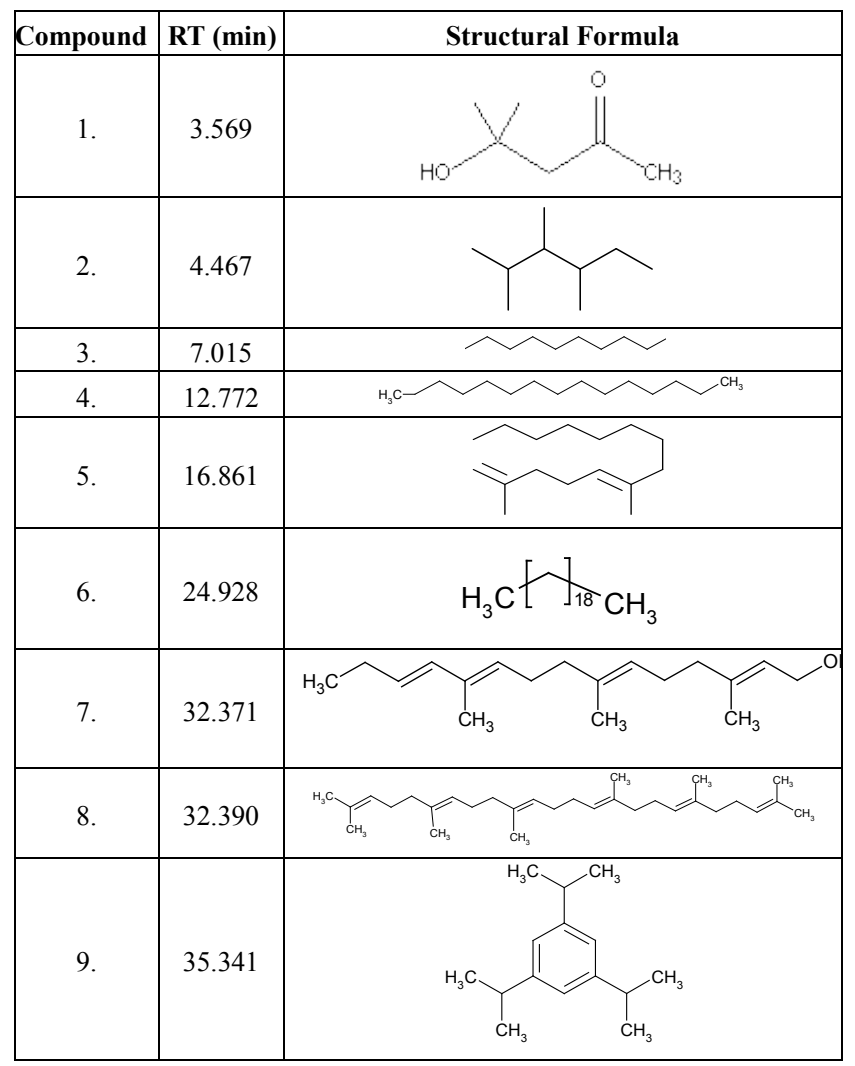

Compound 1 (RT-3.569): Comparison of fragments ions in mass Spectra (MS) of peak (RT 3.569) identified as 4-hydroxy-4-methylpentan-2-one. EI/MS fragment ions $\mathrm{m} / \mathrm{z}$ (percentage base peak): $[\mathrm{M}+] 116,\left[\mathrm{M}^{+}-\mathrm{C}_{2} \mathrm{H}_{3} \mathrm{O}\right] 43(100)$; $\left[\mathrm{M}^{+}-\mathrm{C}_{3} \mathrm{H}_{7} \mathrm{O}\right]$ 58(22); $\left[\mathrm{M}^{+}-\mathrm{C}_{4} \mathrm{H}_{10} \mathrm{O}\right]$ 74(3); $\left[\mathrm{M}^{+}-\mathrm{C}_{3} \mathrm{H}_{5} \mathrm{O}\right]$ 88(3); $\left[\mathrm{M}^{+}-\mathrm{C}_{6} \mathrm{H}_{10} \mathrm{O}\right]$ 98(5); $\left[\mathrm{M}^{+}-\mathrm{C}_{5} \mathrm{H}_{9} \mathrm{O}_{2}\right]$ 101(15).

Compound 2 (RT-4.467): Comparison of fragments ions in mass Spectra (MS) of peak (RT 4.467) identified as 2,3,4trimethylhexane. EI/MS fragment ions $\mathrm{m} / \mathrm{z}$ (percentage base peak): $[\mathrm{M}+] 128 ;\left[\mathrm{M}^{+}-\mathrm{CH}_{3}\right] ; 113(3) ;\left[\mathrm{M}^{+}-\mathrm{C}_{2} \mathrm{H}_{5}\right] 99(10) ;\left[\mathrm{M}^{+}-\right.$ $\left.\mathrm{C}_{4} \mathrm{H}_{9}\right]$; 71(33); $\left[\mathrm{M}^{+}-\mathrm{C}_{6} \mathrm{H}_{13}\right]$ 43(100); $\left[\mathrm{M}^{+}-\mathrm{C}_{5} \mathrm{H}_{11}\right] 57$ (90).

Compound 3 (RT-7.015): Comparison of fragments ions in mass Spectra (MS) of peak (RT 7.015) identified as Decane. EI/MS fragment ions $\mathrm{m} / \mathrm{z}$ (percentage base peak): $[\mathrm{M}+] 142 ;\left[\mathrm{M}^{+}-\mathrm{CH}_{3}\right] 127(3) ;\left[\mathrm{M}^{+}-\mathrm{C}_{2} \mathrm{H}_{5}\right] 113(5) ;\left[\mathrm{M}^{+}-\mathrm{C}_{3} \mathrm{H}_{7}\right]$ 99(5); $\left[\mathrm{M}^{+}-\mathrm{C}_{4} \mathrm{H}_{9}\right]$ 85(30); $\left[\mathrm{M}^{+}-\mathrm{C}_{7} \mathrm{H}_{16}\right]$ 43(100); $\left[\mathrm{M}^{+}-\mathrm{C}_{6} \mathrm{H}_{13}\right]$ 
57(95); $\left[\mathrm{M}^{+}-\mathrm{C}_{18} \mathrm{H}_{17}\right] 29(40)$.

Compound 4 (RT-12.772): Comparison of fragments ions in mass Spectra (MS) of peak (RT 12.772) identified as $\mathrm{n}$-Pentadecane. EI/MS fragment ions $\mathrm{m} / \mathrm{z}$ (percentage base peak): $[\mathrm{M}+]$ 212; $\left[\mathrm{M}-\mathrm{CH}_{3}\right]$ 197(3); $\left[\mathrm{M}^{+}-\mathrm{C}_{2} \mathrm{H}_{5}\right]$ 183(3); $\left[\mathrm{M}^{+}-\mathrm{C}_{3} \mathrm{H}_{7}\right]$ 169(3); $\left[\mathrm{M}^{+}-\mathrm{C}_{4} \mathrm{H}_{9}\right]$ 155(3); $\left[\mathrm{M}^{+}-\mathrm{C}_{11} \mathrm{H}_{23}\right]$ 57(100); $\left[\mathrm{M}^{+}-\mathrm{C}_{12} \mathrm{H}_{25}\right]$ 43(80); [ $\left.\mathrm{M}^{+}-\mathrm{C}_{13} \mathrm{H}_{27}\right]$ 29(35).

Compound 6 (RT-24.928): Comparison of fragments ions in mass Spectra (MS) of peak (RT 24.928) identified as $\mathrm{n}$-Eicosane. EI/MS fragment ions $\mathrm{m} / \mathrm{z}$ (percentage base peak): $[\mathrm{M}+]$ 282; $\left[\mathrm{M}^{+}-\mathrm{C}_{2} \mathrm{H}_{5}\right]$ 267(3); $\left[\mathrm{M}^{+}-\mathrm{C}_{3} \mathrm{H}_{7}\right] 253(3)$; $\left[\mathrm{M}^{+}-\mathrm{C}_{5} \mathrm{H}_{11}\right] \quad 225(3) ; \quad\left[\mathrm{M}^{+}-\mathrm{C}_{17} \mathrm{H}_{35}\right] \quad 57(100) ; \quad\left[\mathrm{M}^{+}-\mathrm{C}_{18} \mathrm{H}_{37}\right]$ 43(73); $\left[\mathrm{M}^{+}-\mathrm{C}_{19} \mathrm{H}_{39}\right] 29(18)$.

Compound 7 (RT-32.371): Comparison of fragments ions in mass Spectra (MS) of peak (RT 32.371) identified as 3, 7,11-Trimethyl-2,6,10,12-pentadecatrien-1-ol. EI/MS fragment ions $\mathrm{m} / \mathrm{z}$ (percentage base peak): $[\mathrm{M}+] 262 ;\left[\mathrm{M}^{+}-\mathrm{OH}\right]$ 244(3); $\left[\mathrm{M}^{+}-\mathrm{C}_{4} \mathrm{H}_{7}\right]$ 207(5); $\left[\mathrm{M}^{+}-\mathrm{C}_{12} \mathrm{H}_{19}\right]$ 99(5); $\left[\mathrm{M}^{+}-\mathrm{CH}_{3} \mathrm{O}\right]$ 231(5); $\left[\mathrm{M}^{+}-\mathrm{C}_{2} \mathrm{H}_{4} \mathrm{O}\right] \quad 218(100) ; \quad\left[\mathrm{M}^{+}-\mathrm{C}_{3} \mathrm{H}_{7} \mathrm{O}\right] ; \quad 203(85) ;$ $\left[\mathrm{M}^{+}-\mathrm{C}_{9} \mathrm{H}_{15}\right] 139(15) ;\left[\mathrm{M}^{+}-\mathrm{C}_{16} \mathrm{H}_{26}\right]$ 44(15); $\left[\mathrm{M}^{+}-\mathrm{C}_{9} \mathrm{H}_{15} \mathrm{O}\right]$ 123(10); $\left[\mathrm{M}^{+}-\mathrm{C}_{10} \mathrm{H}_{17} \mathrm{O}\right]$ 109(15); $\left[\mathrm{M}^{+}-\mathrm{C}_{11} \mathrm{H}_{19} \mathrm{O}\right]$ 95(38).

Compound 8 (RT-32.390): Comparison of fragments ions in mass Spectra (MS) of peak (RT 32.390) identified as 2,6,10,15,19,23-Hexamethyl-2,6,10,14,18,22-tetracosahexa ene. EI/MS fragment ions $\mathrm{m} / \mathrm{z}$ (percentage base peak): $[\mathrm{M}+]$ 410; $\left[\mathrm{M}^{+}-\mathrm{CH}_{3}\right]$ 395(3); $\left[\mathrm{M}^{+}-\mathrm{C}_{4} \mathrm{H}_{7}\right]$ 355(3); $\left[\mathrm{M}^{+}-\mathrm{C}_{16} \mathrm{H}_{27}\right]$ 191(10); $\left[\mathrm{M}^{+}-\mathrm{C}_{26} \mathrm{H}_{43}\right] \quad$ 55(23); $\left[\mathrm{M}^{+}-\mathrm{C}_{25} \mathrm{H}_{41}\right] \quad 69(100)$; $\left[\mathrm{M}^{+}-\mathrm{C}_{5} \mathrm{H}_{9}\right]$ 341(5); $\left[\mathrm{M}^{+}-\mathrm{C}_{6} \mathrm{H}_{11}\right]$ 327(5); $\left[\mathrm{M}^{+}-\mathrm{C}_{9} \mathrm{H}_{15}\right]$ 287(3).

Compound 9 (RT-35.341): Comparison of fragments ions in mass Spectra (MS) of peak (RT 35.341) identified as 1,3,5-Triisopropylbenzene. EI/MS fragment ions $\mathrm{m} / \mathrm{z}$ (percentage base peak): $[\mathrm{M}+]$ 204; $\left[\mathrm{M}^{+}-\mathrm{CH}_{3}\right]$ 189(100); $\left[\mathrm{M}^{+}-\right.$ $\left.\mathrm{C}_{2} \mathrm{H}_{6}\right]$ 174(22); $\left[\mathrm{M}^{+}-\mathrm{C}_{3} \mathrm{H}_{7}\right]$ 161(30); $\left[\mathrm{M}^{+}-\mathrm{C}_{12} \mathrm{H}_{17}\right]$ 43(63).

Compound 5 (RT-16.861): Comparison of fragments ions in mass Spectra (MS) of peak (RT 16.861) identified as 2,6 dimethyl tetra-1,5-decaene. EI/MS fragment ions $\mathrm{m} / \mathrm{z}$ (percentage base peak): $[\mathrm{M}+] 220 ;\left[\mathrm{M}^{+}-\mathrm{CH}_{3}\right] 207(3) ;\left[\mathrm{M}^{+}-\mathrm{C}_{2} \mathrm{H}_{5}\right]$ 193(5); $\left[\mathrm{M}^{+}-\mathrm{C}_{3} \mathrm{H}_{3}\right]$ 181(6); $\left[\mathrm{M}^{+}-\mathrm{C}_{5} \mathrm{H}_{9}\right]$ 153(10); $\left[\mathrm{M}^{+}-\mathrm{C}_{7} \mathrm{H}_{15}\right]$ 123(100); $\left[\mathrm{M}^{+}-\mathrm{C}_{9} \mathrm{H}_{15}\right]$ 99(10); $\left[\mathrm{M}^{+}-\mathrm{C}_{11} \mathrm{H}_{21}\right]$ 69(15); [ $\left[\mathrm{M}^{+}-\right.$ $\left.\mathrm{C}_{14} \mathrm{H}_{25}\right] 29(28)$.

\section{Conclusions}

Acetone extract of C. procera latex investigated through its $n$-hexane fraction with identification of major nine organic compounds with the report of two new compounds 2,6 dimethyl tetra-1,5-decaene and 3, 7,11-Trimethyl-2,6,10, 12-pentadecatrien-1-ol first time from C. procera.

\section{ACKNOWLEDGEMENTS}

We are thankful to the Department of Science and Technology (DST), New Delhi for providing financial support to the project "Development and Standardization of Herbal Anti-malarial Drug". The authors are also gratefully acknowledged the encouragement and support of Director, ARIBAS and Charutar Vidya Mandal (CVM), Vallabh Vidyanagar, Gujarat, India.

\section{REFERENCES}

[1] M D Erdman and B A Erdman, Calotropis procera as a source of plant hydrocarbons. Econ. Bot. 35, 1981, 467-472.

[2] Kumar, Vijay Rani, Ashwani Kumar and A K Gupta, Calotropis procera: A potential bio-energy plant for arid and semi-arid regions. In: Palz, W., J.Spitzer, K.Maniatis, K.Kwant, P.Helm, A.Grassi. (eds) $12^{\text {th }}$ European Biomass conference : Biomass for energy, Industry and climatic protection. WIP Munich. Germany 2002.

[3] Farzin Parabia, I.L.Kothari, and M.H.Parabia, Antibacterial activity of solvent fractions of crude water decoction of apical twigs and latex of Calotropis procera (Ait.) R. Br.". Natural product radiance, 2008, 7(1) 30-34.

[4] Wagner H. and Bladt S. Plant Drug Analysis, Second Edition, Springer, 1996.

[5] I. L. Kothari1, M.H.Parabia, J.H.Patel, Farzin M. Parabia, Smita S. Pathak, Falguni Sheth, Hiren V. Doshi and M.A.Patel, 2010. An Ethnopharmacological Review on Calotropis Species printed in Compendium of Bioactive Natural Products Vol. 3: Efficacy, Safety \& Clinical Evaluation (Part-2) in the book series, Comprehensive Bioactive Natural Products published by Studium Press LLC, USA.

[6] E Haslam, Natural Polyphenols (Vegetable Tannins) as Drugs: Possible Modes of Action. J. Nat. Prod. 1996, 59, 205-2 15.

[7] V E Tyler, Phytomedicines: Back to the Future. J. Nat. Prod. 1999 62, 1589-1592.

[8] J N Seiber, C J Nelson, M S Lee, Cardenolides in the latex and leaves of seven Asclepias species and Calotropis procera. Phytochemistry 21, 1982, 2343-2348.

[9] J A Duke, Handbook of phytochemical constituents of GRAS herbs and other economic plants. Boca Raton, FL. CRC Press. (Dr. Duke's Phytochemical and Ethnobotanical Databases, 1992. [Online Database] (URL: http://www.arsgrin. gov/cgi-bin/duke/farmacy2 .pl). 